\title{
Chaos in the honeycomb optical-lattice unit cell
}

\author{
Max D. Porter and L. E. Reichl \\ Center for Complex Quantum Systems and Department of Physics, The University of Texas at Austin, Austin, Texas 78712, USA
}

(Received 28 October 2015; published 7 January 2016)

\begin{abstract}
Natural and artificial honeycomb lattices are of great interest because the band structure of these lattices, if properly constructed, contains a Dirac point. Such lattices occur naturally in the form of graphene and carbon nanotubes. They have been created in the laboratory in the form of semiconductor 2DEGs, optical lattices, and photonic crystals. We show that, over a wide energy range, gases (of electrons, atoms, or photons) that propagate through these lattices are Lorentz gases and the corresponding classical dynamics is chaotic. Thus honeycomb lattices are also of interest for understanding eigenstate thermalization and the conductor-insulator transition due to dynamic Anderson localization.
\end{abstract}

DOI: 10.1103/PhysRevE.93.012204

\section{INTRODUCTION}

Artificial honeycomb lattices have been realized in a variety of physical systems and support propagation of a variety of waves. Honeycomb lattices composed of carbon atoms, in the form of graphene sheets and carbon nanotubes, occur naturally and support electron matter wave propagation $[1,2]$. Honeycomb lattices that have also been patterned in two-dimensional semiconductor materials (2DEGs) can also support electron wave propagation [3,4]. Honeycomb lattices have been realized in optical lattices and support the propagation of atomic matter waves [5-8]. In addition, photonic crystals have been constructed with honeycomb structure and support interesting effects in electromagnetic wave propagation [9-11]. One reason for the great interest in these honeycomb lattices is the unusual band structure, which if properly constructed, can support a Dirac point.

As we show below, there is another reason why honeycomb lattices might be particularly interesting. Over a wide energy range, gases (composed of electrons, rubidium atoms, or photons) that traverse the honeycomb lattice can be considered to be a Lorentz gas, and the dynamics is classically chaotic. This means that the honeycomb lattice is an ideal system for studying eigenstate thermalization [12-14] or the effects of dynamic Anderson localization on the conduction properties of the lattice [15].

In the sections below, we focus on the dynamics of a dilute gas of rubidium atoms in a honeycomb optical lattice because, as we shall show, this system provides an ideal system for studying the classical-quantum correspondence in lattice systems and, in particular, the effect of chaos on wave propagation in lattices [16,17]. In subsequent sections, we focus only on the unit cell of a honeycomb optical lattice and study the classical-quantum correspondence in the unit cell.

As shown in [18], the honeycomb optical lattice can be formed by an electric field of the form $\mathbf{E}(x, y, t)=$ $\sum_{j=1}^{3} \hat{\epsilon}_{j} E_{0} \cos \left(\mathbf{k}_{j} \cdot \mathbf{r}+\psi_{j}+\omega_{L} t\right)$, where $\omega_{L}=k_{L} c ; c$ is the speed of light; $\mathbf{k}_{1}=0, \quad \mathbf{k}_{2}=\frac{\sqrt{3}}{2} k_{L} \hat{y}-\frac{1}{2} k_{L} \hat{x}$, and $\mathbf{k}_{3}=$ $\frac{\sqrt{3}}{2} k_{L} \hat{y}+\frac{1}{2} k_{L} \hat{x} ; k_{L}$ is the wave vector of the radiation; $\hat{\epsilon}_{j}$ is the polarization of the $j$ th wave; and $\psi_{j}$ denotes the phases of the waves. If we write the electric field in the form $\mathbf{E}(x, y, t)=$
$\mathbf{A}(x, y) \mathrm{e}^{i \omega_{L} t}+\mathbf{A}^{*}(x, y) \mathrm{e}^{-i \omega_{L} t}$, the Hamiltonian describing the center-of-mass motion of rubidium atoms in this radiation field is given by $[19,20]$

$$
H=\frac{p_{x}^{2}}{2 m_{R b}}+\frac{p_{y}^{2}}{2 m_{R b}}+\frac{d^{2}|A(x, y)|^{2}}{\hbar \Delta},
$$

where $d$ is the atomic dipole moment, $\Delta$ is the detuning of the laser frequency from resonance with the atom, and $|A(x, y)|^{2}$ is the intensity of the radiation and determines the potential energy experienced by the rubidium atoms due to their interaction with the laser fields $[19,20]$. It can be written

$$
\begin{aligned}
|A(x, y)|^{2}= & \frac{\left|E_{0}\right|^{2}}{4}\left[3+2 \hat{\epsilon}_{1} \cdot \hat{\epsilon}_{2} \cos \left(k_{L} x+\psi_{21}\right)\right. \\
& +2 \hat{\epsilon}_{1} \cdot \hat{\epsilon}_{3} \cos \left(\frac{\sqrt{3}}{2} k_{L} y+\frac{k_{L} x}{2}+\psi_{31}\right) \\
& \left.+2 \hat{\epsilon}_{2} \cdot \hat{\epsilon}_{3} \cos \left(\frac{\sqrt{3}}{2} k_{L} y-\frac{k_{L} x}{2}+\psi_{32}\right)\right],
\end{aligned}
$$

where $\psi_{i j}=\psi_{i}-\psi_{j}$ denotes the relative phases of the waves. The depth of the potential well $V(x, y)$ is proportional to the laser intensity and is determined by the angle between the polarization vectors of the radiation fields. Each polarization vector $\hat{\epsilon}_{j}$ is perpendicular to the direction of propagation $\mathbf{k}_{j}$ of its radiation field, but $\hat{\epsilon}_{j}$ can be rotated about that axis. The polarization vectors, in spherical coordinates, can be written $\hat{\epsilon}_{1}=\hat{z}$, and $\hat{\epsilon}_{j}=\cos \left(\phi_{j}\right) \sin \left(\theta_{j}\right) \hat{x}+\sin \left(\phi_{j}\right) \sin \left(\theta_{j}\right) \hat{y}+$ $\cos \left(\theta_{j}\right) \hat{z}$ for $j=2,3$, with $\phi_{2}=120^{\circ}$ and $\phi_{3}=60^{\circ}$. Then the coefficients in Eq. (2) can be written $\alpha_{1}=\hat{\epsilon}_{1} \cdot \hat{\epsilon}_{2}, \alpha_{2}=\hat{\epsilon}_{1} \cdot \hat{\epsilon}_{3}$, and $\alpha_{3}=\hat{\epsilon}_{2} \cdot \hat{\epsilon}_{3}=\cos \left(\theta_{2}\right) \cos \left(\theta_{3}\right)+\frac{1}{2} \sin \left(\theta_{2}\right) \sin \left(\theta_{3}\right)$.

Let us now introduce dimensionless variables, $x^{\prime}, y^{\prime}, t^{\prime}, H^{\prime}$, $U^{\prime}, E^{\prime}$, where $x^{\prime}=k_{L} x, y^{\prime}=k_{L} y, t^{\prime}=\omega_{L} t, H=H^{\prime} E_{L}, E=$ $E^{\prime} E_{L}, U=\frac{d^{2}\left|E_{o}\right|^{2}}{2 \hbar \Delta}=U^{\prime} E_{L}, E_{L}=\hbar \omega_{L}=\frac{\hbar^{2} k_{L}^{2}}{2 m_{R b}}, p_{x}=\hbar k_{L} p_{x}^{\prime}$, and $p_{y}=\hbar k_{L} p_{y}^{\prime}$. If we rewrite the Hamiltonian in terms of $x^{\prime}$, $y^{\prime}, t^{\prime}, H^{\prime}, E^{\prime}, U^{\prime}$, then drop the primes and subtract a factor $3 U / 2$, we can write the Hamiltonian in the form

$$
H=p_{x}^{2}+p_{y}^{2}+U V(x, y)=E-\frac{3}{2} U,
$$


where

$$
\begin{gathered}
V(x, y)=\alpha_{1} \cos (x)+\alpha_{2} \cos \left(\frac{\sqrt{3} y}{2}+\frac{x}{2}-\pi\right) \\
+\alpha_{3} \cos \left(\frac{\sqrt{3} y}{2}-\frac{x}{2}-\pi\right),
\end{gathered}
$$

and we have made a particular choice of the phases $\psi_{21}=$ $0, \psi_{31}=\psi_{32}=-\pi$ to give a convenient orientation of the lattice unit cell with respect to the coordinate frame. This Hamiltonian neglects the interactions between the rubidium atoms, which form a dilute gas, and only accounts for the atom-radiation interaction. For gas comprised of rubidium atoms, $m_{\mathrm{Rb}}=86.909 \mathrm{u}$ (the mass of ${ }^{87} \mathrm{Rb}$ ). The recoil energy of rubidium is $E_{L}=2.156 \times 10^{-30} \mathrm{~J}$ so $\omega_{L}=2.044 \times 10^{4} \mathrm{rad} / \mathrm{s}$.

The Hamiltonian in Eqs. (3) and (4) has an important scale invariance. Let us change the intensity of the laser radiation so that the new intensity $\tilde{U}$ is proportional to the old intensity $U$ with $\tilde{U}=\beta U$. For the case $\beta>1(\beta<1)$ this will increase (decrease) the height of the optical lattice potential energy and change the energy scale of the dynamics. However, let us now make the following changes in the variables: $E=\beta \tilde{E}$, $H=\beta \tilde{H}, p_{x}=\sqrt{\beta} \tilde{p}_{x}, p_{y}=\sqrt{\beta} \tilde{p}_{y}$, and $t=\tilde{t} / \sqrt{\beta}$. Then the Hamiltonian in Eq. (3) takes the form

$$
\tilde{H}=\tilde{p}_{x}^{2}+\tilde{p}_{y}^{2}+U V(x, y)=\tilde{E}-\frac{3}{2} U .
$$

Also, Hamilton's equations remain unchanged, except they are expressed in terms of the variables $\left\{\tilde{p}_{x}, \tilde{p}_{y}, x, y, \tilde{t}\right\}$ rather than $\left\{p_{x}, p_{y}, x, y, t\right\}$, and $U$ remains unchanged. This scaling property is very important for the quantum-classical correspondence of the system because action variables associated with periodic orbits scale as $\sqrt{\beta}$. Since semiclassical quantization stipulates that action is quantized in units of Planck's constant $h$ [21], it means that the number of quantum states that a given periodic orbit in the classical phase space can support increases as $\sqrt{\beta}$. As we scale the laser intensity, the spatial scale and identity of dynamical structures such as periodic orbits and Kolmogorov-Arnold-Moser (KAM) islands do not change, but their energy and the number of quantum states they support does change. Thus, this is a perfect system for studying the quantum-classical correspondence for wave motion through a periodic lattice. In subsequent sections, we will analyze the dynamics for $U=20$, a value attainable in current experiments [22] and a value for which localized states can exist in the graphene optical lattice.

When the polarizations of all three waves are parallel, for example, $\theta_{2}=\theta_{3}=0$ so $\alpha_{1}=\alpha_{2}=\alpha_{3}=1$, the optical lattice has a perfect hexagonal structure similar to graphene. In Fig. 1(a), we show a contour plot of the potential energy $V(x, y)$ for the case $\alpha_{1}=\alpha_{2}=\alpha_{3}=1$. We have indicated the lattice unit cell of graphene by the dashed lines. The primitive vectors for the graphene unit cell are $\mathbf{a}_{1}=2 \pi \hat{e}_{x}+\frac{2 \pi}{\sqrt{3}} \hat{e}_{y}$ and $\mathbf{a}_{2}=2 \pi \hat{e}_{x}-\frac{2 \pi}{\sqrt{3}} \hat{e}_{y}$, and the lattice constant is $\left|\mathbf{a}_{1}\right|=\left|\mathbf{a}_{2}\right|=$ $\frac{4 \pi}{\sqrt{3}}$. The unit cell has an area of $\Omega=\frac{8 \pi^{2}}{\sqrt{3}}$.

It is interesting to consider what happens to the lattice when the laser polarizations are not perfectly aligned. In Fig. 1(b), we show a contour plot of the lattice potential energy for the case $\theta_{2}=30^{\circ}$ and $\theta_{3}=60^{\circ}$, so that $\alpha_{1}=\frac{\sqrt{3}}{2}, \alpha_{2}=\frac{1}{2}$, and (a)

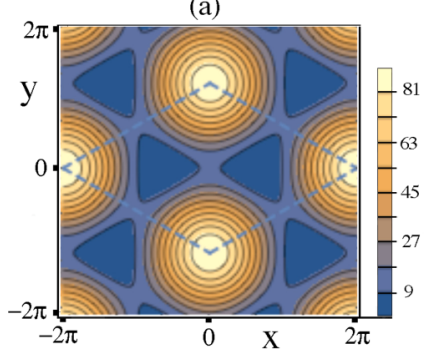

(b)

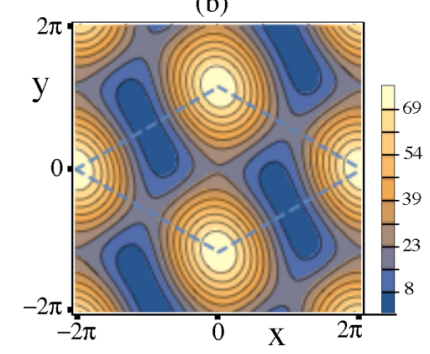

FIG. 1. (a) Contour plot of the potential energy for $U=20$ and $\theta_{1}=\theta_{2}=\theta_{3}=0^{\circ}$ (graphenelike honeycomb). (b) Contour plot of the potential energy for $U=20$ and $\theta_{1}=0^{\circ}, \theta_{2}=30^{\circ}$, and $\theta_{3}=60^{\circ}$ (twisted honeycomb).

$\alpha_{3}=\frac{3 \sqrt{3}}{8}$. All the maxima, and the five saddle points of the unit cell, remain in the same position. However, the interior of the unit cell becomes twisted relative to that of graphene and the position of the two potential energy minima inside the unit cell changes.

\section{CLASSICAL DYNAMICS}

The classical dynamics of the optical lattice gives important insight into the behavior of the quantum system. The "unit cell" for the classical dynamics is one-half of the graphene unit cell described above-for example, the equilateral triangle on the right half of the graphene unit cell-which we shall call the half cell. The structure of the classical dynamics repeats itself in each of these half cells. In subsequent sections we will focus the classical dynamics in the right half cell. The classical half cell forms a symmetric equilateral triangle and has potential energy maxima at each corner of the triangle. It has a saddle at the center of each boundary line, and it has a potential energy minimum at its center.

We can determine the location of the dominant fixed points of the lattice from Hamilton's equations, which can be written

$$
\begin{aligned}
\frac{d p_{x}}{d t}=U \alpha_{1} \sin (x)-\frac{1}{2} U \alpha_{2} \sin \left(\frac{x}{2}+\frac{\sqrt{3} y}{2}\right) \\
-\frac{1}{2} U \alpha_{3} \sin \left(\frac{x}{2}-\frac{\sqrt{3} y}{2}\right), \\
\frac{d p_{y}}{d t}=-\frac{\sqrt{3}}{2} U \alpha_{2} \sin \left(\frac{x}{2}+\frac{\sqrt{3} y}{2}\right) \\
+\frac{\sqrt{3}}{2} U \alpha_{3} \sin \left(\frac{x}{2}-\frac{\sqrt{3} y}{2}\right), \\
\frac{d x}{d t}=2 p_{x}, \frac{d y}{d t}=2 p_{y} .
\end{aligned}
$$

Fixed points are points for which $\dot{p}_{x}=\dot{p}_{y}=\dot{x}=\dot{y}=0$. There are several fixed points of these equations that are independent of the values of $U$ and $\alpha_{j}$, as can be seen in Figs. 1(a) and 1(b). These include the potential energy maxima and the saddle points. The potential energy maxima occur at the corners of the half cell $\left(x_{m x}=2 \pi, y_{m x}=0\right)$ and $\left(x_{m x}=\right.$ $\left.0, y_{m x}= \pm \frac{2 \pi}{\sqrt{3}}\right)$, and have energy $E_{m x}=\left(\alpha_{1}+\alpha_{2}+\alpha_{3}\right) U+$ $\frac{3 U}{2}$. There are three saddle points associated to the half cell. The saddle point located at $\left(x_{s d 1}=0, y_{s d 1}=0\right)$ has energy 
$E_{s d 1}=\left(\alpha_{1}-\alpha_{2}-\alpha_{3}\right) U+\frac{3 U}{2}$. The saddle points located at $\left(x_{s d \pm}=\pi, y_{s d \pm}= \pm \frac{\pi}{\sqrt{3}}\right)$ have energy $E_{s d \pm}=$ $\left(-\alpha_{1} \pm \alpha_{2} \mp \alpha_{3}\right) U+\frac{3 U}{2}$. For graphene $\left(\alpha_{1}=\alpha_{2}=\alpha_{3}=1\right)$, the saddle points have equal energy $E_{s d 1}=E_{s d \pm}=10$. For other values of $\alpha_{i}$, the saddle points, which control the flow of trajectories through the lattice, have different energy.

There is one potential energy minimum inside the half cell. For the case of graphene $\left(\alpha_{1}=\alpha_{2}=\alpha_{3}=1\right)$, the fixed point at the potential energy minimum is located at $\left(x_{m n}=\frac{2 \pi}{3}, y_{m n}=\right.$ 0 ) and it has energy $E_{m n}=0$. For the lattice shown in Fig. 1(b), the fixed point at the potential energy minimum is located at $\left(x_{m n}=2.53, y_{m n}=0.45\right)$ and the potential energy is $E_{m n}=$ -1.08 .

For the graphenelike optical lattice, the classical dynamics for the energy interval below the saddle point energy $(0 \leqslant E \leqslant$ 10) has a mixed phase space. We can visualize the dynamics with Poincarè surfaces of section [23]. Each half cell has three straight lines that are local minima of the potential energy (minimum potential energy "trenches"). They start at the saddle points and end at the potential energy minimum at the center of the half cell. We can use Birkhoff coordinates to obtain Poincarè surfaces of section (SOSs) along each of these trenches, and they will be identical due to the symmetry of the lattice. All SOSs we show here are plots of $p_{x}$ versus $x$, plotted each time the trajectory crosses the line $y=0$ with positive $p_{y}$. While only the portion of this line $0<x<2 \pi / 3$ is a trench, we can extend the line $y=0$ across the full width of the half cell to a more extended SOS. In Fig. 2. we show the progression of the dynamics for surfaces of section along the $y=0$ trench in the unit half cell. In Fig. 2(a), we show the SOS for energy $E=5.338$. It is dominated by a KAM torus. All SOSs in an approximate energy range $0<E \leqslant 5.5$ have this same large-scale structure and simply grow in size as the energy increases. Above energy $E \approx 5.5$ the chaos begins to spread, as can be seen in Fig. 2(b), and as we reach the saddle point energy the phase space is dominated by chaos. It is interesting to note that these SOSs are invariant under changes in $U$ due to the scaling property discussed earlier.

Figures 2(a) and 2(b) show the dominant stable and unstable periodic orbits of the SOS. The corresponding configuration space orbits are shown in Fig. 3. There are two dominant period-one stable periodic orbits in Fig. 2(a), located at $\left(\frac{p_{x}}{\sqrt{E}}=\right.$ $0, x=1.55)$ and $\left(\frac{p_{x}}{\sqrt{E}}=0, x=2.74\right)$. These are due to the configuration space periodic orbit in Fig. 3(a) and its time-reversed twin. This periodic orbit, and its time reversed twin, each undergo a bifurcation at energy $E=9.09$ and give rise to the pairs of small stable islands shown in the middle and on the right side of Fig. 2(c). The configuration space version of the bifurcated orbit is shown in Fig. 3(b). There are three unstable period-one periodic orbits in Fig. 2(a), located at $\left(\frac{p_{x}}{\sqrt{E}}= \pm 0.66, x=1.60\right)$ and $\left(\frac{p_{x}}{\sqrt{E}}=0, x=2.38\right)$ coming from the three independent unstable periodic orbits in Fig. 3(c). If one moves slightly off of these unstable periodic orbits, the trajectory lies in the chaotic sea and undergoes a random walk through the phase space, as shown in Fig. 3(d). This random walk occurs mostly in the neighborhood of the periodic orbits, but the trajectory eventually visits the neighborhood of all three periodic orbits.

When the phase space is predominantly integrable, and large regions of KAM tori exist, we can hope to use

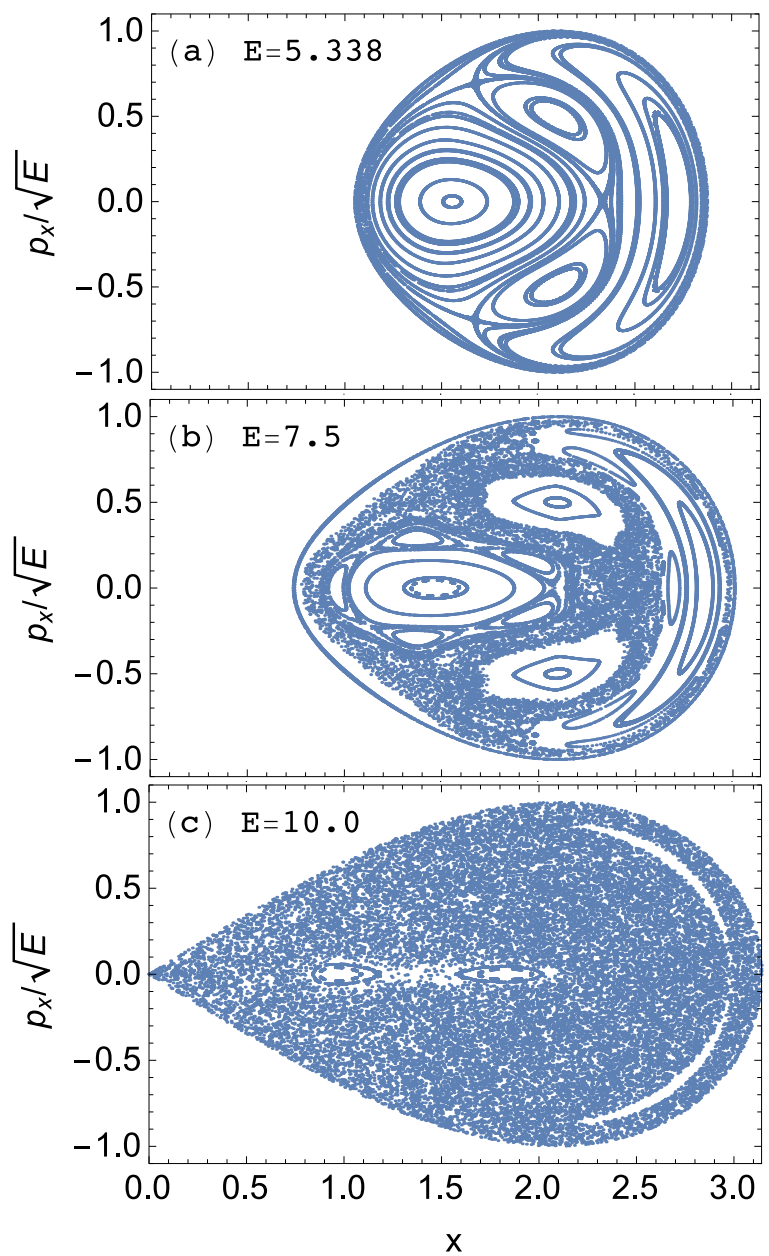

FIG. 2. Surfaces of section of ( $\left.p_{x}, x\right)$ for $y=0$ and $p_{y}>0$ below and at the saddle energy: (a) $E=5.338$, (b) $E=7.5$, and (c) $E=$ 10.0.

semiclassical quantization to determine the existence of quantum states in this region. The semiclassical quantization condition allows us to determine if the potential well below the saddle can hold a quantum state. To hold a quantum state, a classical orbit must satisfy the action relationship $J=n h$, where $\mathrm{J}$ is the action (equal to the area enclosed by the orbit in phase space), $h$ is Planck's constant, and $n$ is an integer. The dominant period-one stable periodic orbits satisfy this condition at energy $E \approx 5.473$. This periodic orbit, and its time-reversed twin, appear to allow the creation of a standing-wave bound state of the lattice. (This energy differs only slightly from the symmetric quantum ground state shown in Fig. 7 and with $E \approx 5.30$.) Above these energies, the growing regions of chaos, and destruction of action as a good quantum number, preclude the use of semiclassical quantization [23].

For energies above the saddle point energy, there is chaotic flow throughout the lattice. In the half cell, trajectories can enter and leave the half cell through all three saddle points. The mixing of the chaotic flow from the three saddle points can be seen in Fig. 4. The saddle points themselves have energy $E=10$. However, the saddle provides an unstable period-one orbit in the SOS for energies $10 \leqslant E<90$, where 

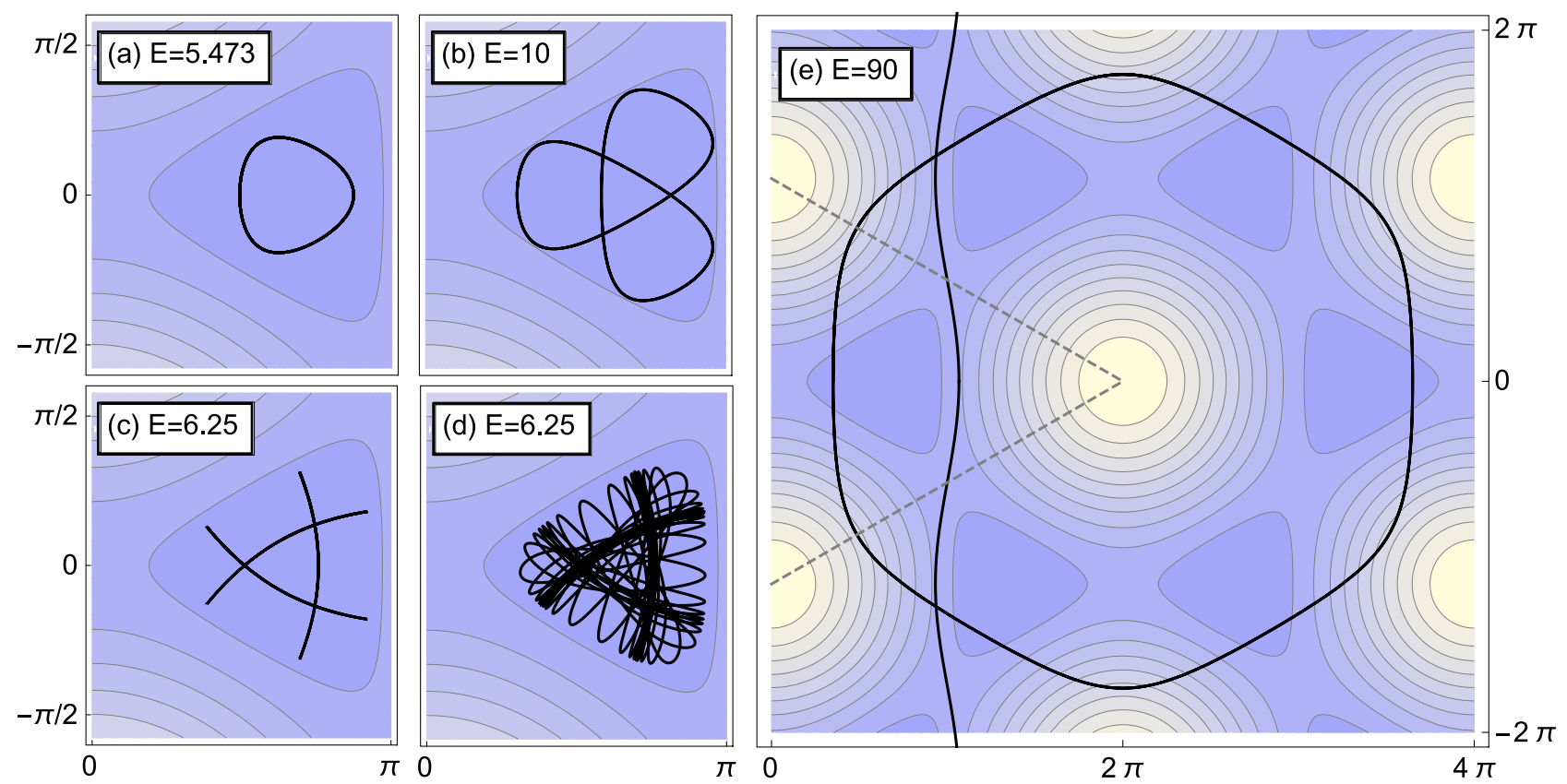

FIG. 3. Configuration space plots of key periodic orbits ( $x$ axis is horizontal and $y$ axis is vertical). (a) Dominant periodic orbit below the saddle point energy, $E=5.473$. (b) Bifurcated orbit in (a) but at energy $E=10$. (c) Three unstable periodic orbits at $E=6.25$. (d) Trajectory in chaotic sea at $E=6.25$. (e) Two dominant periodic orbits at high energy, $E=90$.

$E=90$ is the energy of the potential peak. In Fig. 4(a), we show the unstable manifold coming off the saddle at $(x=0, y=0)$ and energy $E=12.5$. The saddles have a stable direction, along which the orbits oscillate periodically, and an unstable direction in which the orbits (roll down the hill) move away from the saddle point with growing speed. An unstable manifold at energy $E=12.5$ has an oscillatory motion as it moves away from the fixed point that allows for a clean SOS. The unstable manifold is area preserving and crosses and recrosses its own saddle region as it evolves. It appears to fill the chaotic region of the phase space [see Fig. 4(c)]. In Fig. 4(b) we show the same surface of section but now include the flow of the stable manifolds that originate from the saddles at $\left(x=\pi, \pm \frac{\pi}{\sqrt{3}}\right)$. The unstable manifolds from all three saddle points mix together and flow back and forth across the saddle at $(x=0, y=0)$. If we did SOSs along the minimum potential energy trenches that start from the saddle points at $\left(x=\pi, \pm \frac{\pi}{\sqrt{3}}\right)$, we would see identical behavior. This gives a clear indication of the highly mixing behavior of the chaotic flow through the optical lattice. Indeed, trajectories in the chaotic sea undergo a random walk through the optical lattice, even though the Newtonian dynamics is completely deterministic.

For energies above the saddle point energy, $10 \leqslant E \leqslant 90$, atoms moving though the optical lattice "see" potential barriers arranged like that of a Lorenz gas. For the energy interval $E_{s d}=10$ to about $E \approx 32$, the SOS in the half cell is dominated by chaos, although small stable islands do appear in the SOS. For the energy range $32<E<55$, no stable islands appear in the SOS, as can be seen from the SOS in Fig. 5(a), and the dynamics may well be a true $\mathrm{K}$ flow, in analogy to the behavior found in [24]. Above $E \approx 55$, three stable fixed points emerge in the SOSs. At slightly higher energies, three additional, but much smaller, stable fixed points appear in the SOS. These six stable fixed points begin to dominate the SOS at energy $E_{\text {peak }}=90$, as can be seen in Fig. 5(b). They are the result of periodic orbits that exist in the configuration space at these higher energies. The two configuration space periodic orbits that give rise to the six dominant stable fixed points in Fig. 5(b) are shown in Fig. 3(e). As we go higher in energy, larger regions of chaos appear and then gradually disappear at very high energies. Indeed, there still is significant chaos at $E=360$. This complex structure of the phase space, for energies above $E=90$, is the result of higher-order resonances that exist at these high energies.

The potential energy for the honeycomb half cell has some similarities to several other systems whose dynamics have been well studied. At low energy, the SOS for the half cell is similar to that of the Henon-Heiles system [25]. The Henon-Heiles system (developed to study the dynamics of galaxies) has three symmetrically placed potential energy hills whose upward slopes always have positive curvature. The orbits shown in Fig. 2(a) are almost identical to low energy SOSs from the Henon-Heiles system, and result from trajectories bouncing between half-cell potential energy hills in an energy region where the hills have upward positive curvature. The Henon-Heiles system differs in two important respects. It doesn't have saddle points between the peaks. In the Henon-Heiles system, once a trajectory passes between the potential energy hills, it travels out over an increasingly negative potential energy landscape. Another system that has similarities to the honeycomb half cell is the triple hill potential studied by Jung and Richter [24]. The triple hill potential has saddle points between the potential hills, like the honeycomb half cell. However, the potential well under the saddle is very shallow, with a depth below the saddle of about $5 \%$ of the 


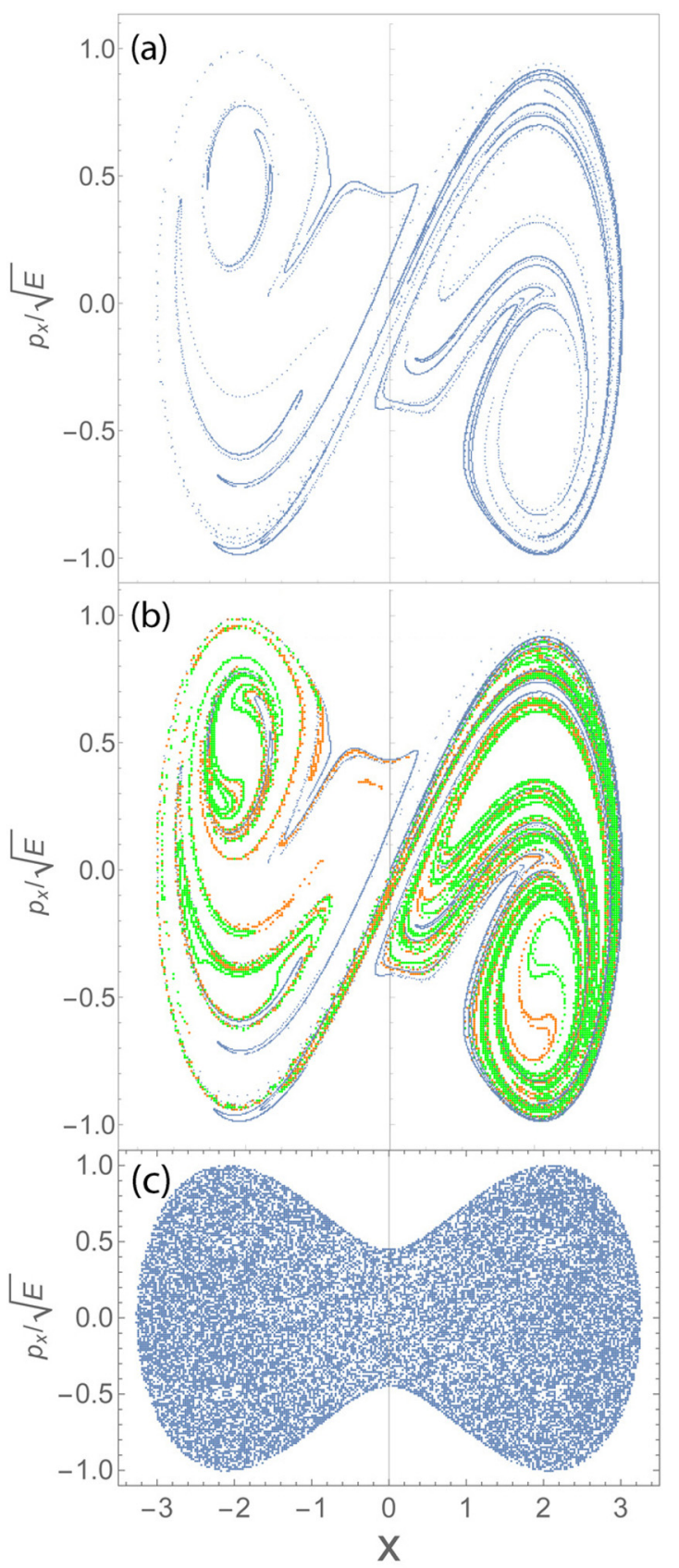

FIG. 4. (a) SOS of $\left(p_{x}, x\right)$ for $y=0$ and $p_{y}>0$, of the unstable manifold that emerges from the saddle at $(x=0, y=0)$ for energy $E=12.5$. (b) SOS of $\left(p_{x}, x\right)$ for $y=0$ and $p_{y}>0$ containing the unstable manifolds coming from all three saddles in the half cell for $E=12.5$. The unstable manifold coming from the central saddle at $(x=0, y=0)$ is in blue (dark gray, small dots), from the saddle at $(\pi, \pi / \sqrt{3})$ is in orange (medium gray, big dots), and from the saddle at $(\pi,-\pi / \sqrt{3})$ is in green (light gray, big dots). (c) Phase space SOS of $\left(p_{x}, x\right)$ for $y=0$ and $p_{y}>0$ for a range of initial conditions in the half cell for energy $E=12.5$.

potential height (for the honeycomb half cell it is about 11\%), so it is less likely to support a quantum bound state. The upward slope of the triple hill potential, like that of the honeycomb half cell, changes from positive to negative part-way up. Jung and

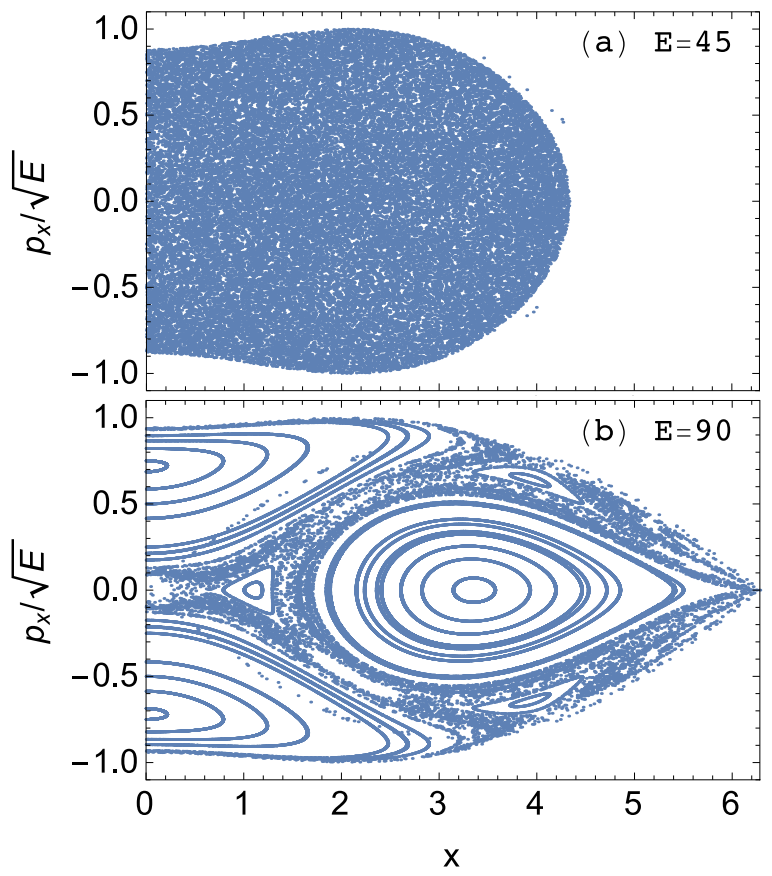

FIG. 5. SOS of $\left(p_{x}, x\right)$ for $y=0$ and $p_{y}>0$ for (a) $E=45$ and (b) $E=90$.

Richter [24] have analyzed the structure of periodic orbits in the triple hill system and show that there is an energy interval, below the potential energy maximum, where all periodic orbits are unstable, indicating that, in that energy interval, the motion is completely chaotic, i.e., it is a $\mathrm{K}$ flow and similar to the dynamics of a truly chaotic scattering system consisting of three hard disks [26-29]. (The scattering dynamics of three hard disks is a $\mathrm{K}$ flow at all energies.) Thus, in many respects, the behavior of the triple hill system is very similar to that of the honeycomb half cell.

\section{QUANTUM DYNAMICS}

Each unit cell of graphene consists of two half cells of the classical system. If we consider only the dynamics of the unit cell, the energy eigenstates can be written

$$
u_{E}(\mathbf{r})=\frac{\sqrt{3}}{8 \pi^{2}} \sum_{n_{1}, n_{2}=-\infty}^{\infty} A_{E ; n_{1}, n_{2}} \mathrm{e}^{i\left(n_{1} \mathbf{b}_{1}+n_{2} \mathbf{b}_{2}\right) \cdot \mathbf{r}},
$$

where $\mathbf{b}_{1}=\frac{1}{2} \hat{e}_{x}+\frac{\sqrt{3}}{2} \hat{e}_{y}$ and $\mathbf{b}_{2}=\frac{1}{2} \hat{e}_{x}-\frac{\sqrt{3}}{2} \hat{e}_{y}$ are the reciprocal lattice vectors. The eigenvalue equation in a unit cell then takes the form

$$
\hat{H} u_{E}(\mathbf{r})=\left(-\frac{\hbar^{2}}{2 m} \nabla^{2}+V(\mathbf{r})\right) u_{E}(\mathbf{r})=E u_{E}(\mathbf{r}) .
$$

The boundary condition for $u_{\mathbf{k}}(\mathbf{r})$ is $u_{\mathbf{k}}\left(\mathbf{r}+\mathbf{a}_{i}\right)=u_{\mathbf{k}}(\mathbf{r}), i=$ 1,2 . We can write $u_{\mathbf{k}}(\mathbf{r})$ as

$$
u_{\mathbf{k}}(\mathbf{r})=\frac{1}{\sqrt{\Omega}} \sum_{\mathbf{G}} A_{\mathbf{G}} e^{i \mathbf{G} \cdot \mathbf{r}},
$$

where $\quad \mathbf{G}=n_{1} \mathbf{b}_{1}+n_{2} \mathbf{b}_{2} \quad\left(n_{1}, n_{2}=0, \pm 1, \pm 2, \ldots\right.$, $\pm \infty)$, and $A_{\mathbf{G}}$ are coefficients to be determined. The basis set 

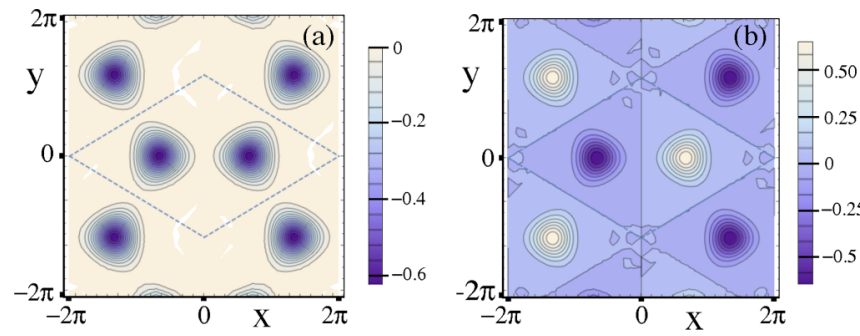

FIG. 6. The wave functions for the lowest energy eigenstates in the unit cell: (a) $E=5.30$, (b) $E=5.31$.

has been normalized so that

$$
\frac{1}{\Omega} \int_{\Omega} d \mathbf{r} e^{-i\left(\mathbf{G}^{\prime}-\mathbf{G}\right) \cdot \mathbf{r}}=\delta_{\mathbf{G}^{\prime}, \mathbf{G}} .
$$

After we substitute Eq. (9) into Eq. (8), then, with the aid of Eq. (10), we can write the Schrödinger equation in matrix form:

$$
\sum_{\mathbf{G}^{\prime}}\left[\frac{\hbar^{2}}{2 m} G^{2} \delta_{\mathbf{G}, \mathbf{G}^{\prime}}+V_{\mathbf{G}, \mathbf{G}^{\prime}}\right] A_{\mathbf{G}^{\prime}}=E_{\mathbf{k}} A_{\mathbf{G}} .
$$

In Fig. 6 we show the wave functions (to within an overall phase factor) of the two lowest energy eigenstates (with energies $E=5.30$ and $E=5.31$ ) of the unit cell (the plots obtained for $-7 \leqslant n_{1} \leqslant 7$ and $-7 \leqslant n_{2} \leqslant 7$ ). The ground state of the lattice $(E=5.30)$ is symmetric in the unit cell. The first excited state $(E=5.31)$ is antisymmetric in the unit cell. These two lowest states are the only eigenstates with energy below the saddle point energies and may be thought to correspond to the $\pi$ bonds of graphene. The next higher energy eigenstates have energies just above the saddle energies and form two degenerate pairs of states. The wave functions for
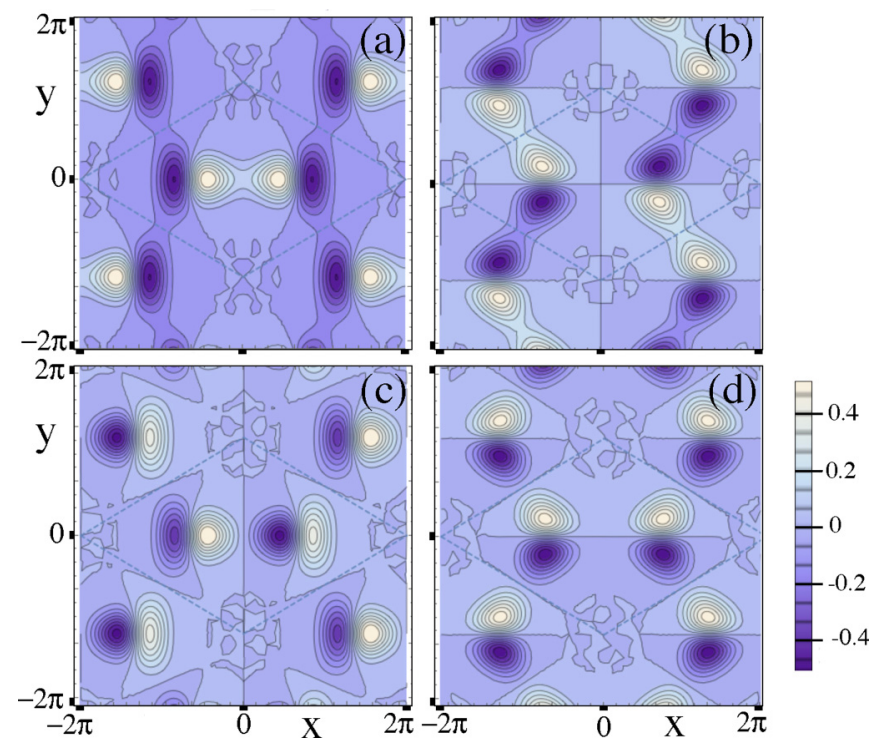

FIG. 7. The wave functions for the third through sixth energy eigenstates. They form degenerate pairs. (a) and (b) have energy $E=10.09$, which is just above the saddle. (c) and (d) have energy $E=10.25$.

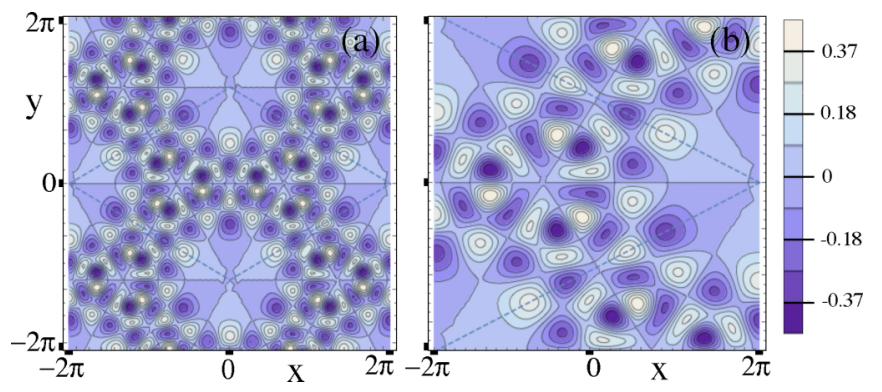

FIG. 8. (a) Wave function for an energy eigenstate in the chaotic sea at energy $E=57.8$. The dashed line indicates the unit cell. (b) The same state with focus on the probability amplitude in the half cell.

the degenerate states with energy $E=10.09$ are shown in Figs. 7(a) and 7(b). This pair of degenerate states is clearly associated with the saddle points. The wave functions for the degenerate states with energy $E=10.25$ are shown in Figs. 7(c) and 7(d). These states appear to be excited states of the potential wells in the unit cell.

In Fig. 8(a), we show an energy eigenstate for energy $E=57.79$ where the phase space is in the chaotic regime. The unit cell is indicated by the dashed lines. For the laser intensity we are considering, $U=20$, the system is far from the semiclassical regime. However, some of the signatures of chaos can be seen. The state is symmetric about the line $x=0$ and it is antisymmetric about the line $y=0$. In Fig. 8(b), we focus on the half cell. There is a sixfold symmetry for this state, but within each of the six triangles in the half cell, we begin to see the irregular nodal patterns characteristic of eigenstates in a chaotic system. As we increase the laser intensity, the energy of these states will scale upward in the manner described earlier, and we expect to see a denser irregular pattern of nodal lines, similar to chaotic billiards like the stadium or the Sinai billiard [23].

\section{CONCLUSIONS}

We have analyzed the classical and quantum dynamics of the unit cell of a honeycomb optical lattice. The honeycomb optical lattice is of particular interest because, with the proper scaling, the dynamical structure of the phase space remains unchanged as the energy of the system is changed. This means that it is possible to go from the quantum regime to the semiclassical regime, without changing the basic dynamics of the system. Below the saddle, classical trajectories are localized. Above the saddle, the system consists of an array of circular barriers, and the dynamics of particles (electrons, atoms, photons) confined to the lattice is that of a Lorentz gas. The particle dynamics over a wide energy range is chaotic.

For a laser intensity commonly found in optical lattice experiments, we have found that the unit cell of the honeycomb lattice contains a ground state that is symmetric and has energy below the low energy saddle (and an antisymmetric state with 
only slightly higher energy). These two low energy states are well separated in energy from all other energy eigenstates, all of which lie above the saddle.

This system, because it can be scaled from the quantum regime to the semiclassical regime without changing the qualitative structure of the dynamics, provides an important system for studying the effects of lattice dynamics on the Dirac point, the validity of the "eigenstate thermalization hypothesis," and the possible occurrence of dynamic Anderson localization as a mechanism for the conductor-insulator transition in the lattice.

\section{ACKNOWLEDGMENTS}

The authors thank the Robert A. Welch Foundation (Grant No. F-1051) for support of this work. We also thank Christof Jung for helpful comments.
[1] R. Saito, G. Dresselhaus, and M. S. Dresselhaus, Physical Properties of Carbon Nanotubes (Imperial College Press, London, UK, 1998).

[2] M. I. Katsnelson, Graphene: Carbon in Two Dimensions (Cambridge University Press, Cambridge, UK, 2012).

[3] M. Gibertini, A. Singha, V. Pellegrini, M. Polini, G. Vignale, A. Pinczuk, L. N. Pfeiffer, and K. W. West, Phys. Rev. B 79, 241406(R) (2009).

[4] S. Goswami, M. A. Aamir, C. Siegert, M. Pepper, I. Farrer, D. A. Ritchie, and A. Ghosh, Phys. Rev. B 85, 075427 (2012).

[5] G. Grynberg, B. Lounis, P. Verkerk, J.-Y. Courtois, and C. Salomon, Phys. Rev. Lett. 70, 2249 (1993).

[6] C. Wu, D. Bergman, L. Balents, and S. Das Sarma, Phys. Rev. Lett. 99, 070401 (2007).

[7] C. Wu and S. Das Sarma, Phys. Rev. B 77, 235107 (2008).

[8] L. Tarruell et al., Nature (London) 483, 302 (2012).

[9] F. D. M. Haldane and S. Raghu, Phys. Rev. Lett. 100, 013904 (2008).

[10] R. A. Sepkhanov, Ya. B. Bazaliy, and C. W. J. Beenakker, Phys. Rev. A 75, 063813 (2007).

[11] R. A. Sepkhanov, J. Nilsson, and C. W. J. Beenakker, Phys. Rev. B 78, 045122 (2008).

[12] J. M. Deutsch, Phys. Rev. A 43, 2046 (1991).

[13] M. Srednicki, Phys. Rev. E 50, 888 (1994).

[14] M. Rigol and M. Srednicki, Phys. Rev. Lett. 108, 110601 (2012).
[15] D. M. Basko, I. L. Aleiner, and B. L. Altshuler, Ann. Phys. 321, 1126 (2006).

[16] G. A. Luna-Acosta, K. Na, L. E. Reichl, and A. Krokhin, Phys. Rev. E 53, 3271 (1996).

[17] Y. Boretz and L. E. Reichl, Phys. Rev. E 91, 042901 (2015).

[18] K. L. Lee, B. Gremaud, R. Han, B.-G. Englert, and C. Miniatura, Phys. Rev. A 80, 043411 (2009).

[19] R. Graham, M. Schlautmann, and P. Zoller, Phys. Rev. A 45, R19 (1992).

[20] Benjamin P. Holder and L. E. Reichl, Phys. Rev. A 76, 013420 (2007).

[21] M. Born, The Mechanics of the Atom (Frederick Ungar Publishing Co., New York, 1960).

[22] M. Greiner, O. Mandel, T. Esslinger, T. W. Hansch, and I. Bloch, Nature (London) 415, 39 (2002).

[23] L. E. Reichl, The Transition to Chaos, 2nd ed. (Springer-Verlag, New York, 2004).

[24] C. Jung and P. H. Richter, J. Phys. A 23, 2847 (1990).

[25] M. Henon and C. Heiles, Astron. J. 69, 73 (1964).

[26] B. Eckhardt, J. Phys. A 20, 5971 (1987).

[27] P. Gaspard and S. A. Rice, J. Chem. Phys. 90, 2225 (1989); 90, 2242 (1989); 90, 2255 (1989).

[28] T. Geisel et al., Z. Phys. B 71, 117 (1988).

[29] P. Gaspard and F. Baras, Phys. Rev. E 51, 5332 (1995). 